\title{
A note on Sum, Difference, Product and Ratio of Kumaraswamy Random Variables
}

\author{
Avishek Mallick \\ Department of Mathematics, Marshall University, \\ Huntington, West Virginia 25755, USA \\ mallicka@marshall.edu \\ Indranil Ghosh \\ Department of Mathematics and Statistics, University of North Carolina, \\ Wilmington, North Carolina 28403, USA \\ ghoshi@uncw.edu \\ G. G. Hamedani \\ Department of Mathematics, Statistics and Computer Science, Marquette University, \\ Milwaukee, Wisconsin 53201, USA \\ gholamhoss.hamedani@marquette.edu
}

Received 14 December 2016

Accepted 15 September 2017

\begin{abstract}
Explicit expressions for the densities of $S=X_{1}+X_{2}, D=X_{1}-X_{2}, P=X_{1} X_{2}$ and $R=X_{1} / X_{2}$ are derived when $X_{1}$ and $X_{2}$ are independent or sub-independent Kumaraswamy random variables. The expressions appear to involve the incomplete gamma functions. Some possible real life scenarios are mentioned in which such quantities might be of interest.

Keywords: Ratio of random variables; product of random variables; Kumaraswamy distribution; subindependence.
\end{abstract}

2000 Mathematics Subject Classification: 62E10, 62E15

\section{Introduction}

Kumaraswamy (1980) [3] introduced a two parameter absolutely continuous distribution which compares extremely favorably, in terms of simplicity, with the beta distribution. The Kumaraswamy distribution on the interval $(0,1)$, has its probability density function (pdf) with two shape parameters $a>0$ and $b>0$ defined by

$$
f(x)=a b x^{a-1}\left(1-x^{a}\right)^{b-1} I(0<x<1)
$$

and its cumulative distribution function (cdf) is given by

$$
F(x)=1-\left(1-x^{a}\right)^{b} .
$$


If a random variable $X$ has pdf given in (1.1) then we will write $X \sim K(a, b)$.

The density function in (1.1) has similar properties to those of the beta distribution but has some advantages in terms of tractability. The Kumaraswamy pdf is unimodal, uniantimodal, increasing, decreasing or constant depending (similar to the beta distribution) on the values of the parameters. It has some basic properties of the beta distribution: $a>1$ and $b>1$ (unimodal); $a<1$ and $b<1$ (uniantimodal); $a>1$ and $b \leq 1$ (increasing); $a \leq 1$ and $b>1$ (decreasing); $a=b=1$ (constant). For a detailed survey of properties of the Kumaraswamy distribution, the reader is referred to Jones (2009) [2]. This distribution has a close relation with beta and generalized beta (first kind) as listed below:

- If $X \sim \operatorname{Beta}(1, b)$ then $X \sim \mathrm{K}(1, b)$

- If $X \sim \operatorname{Beta}(a, 1)$ then $X \sim \mathrm{K}(a, 1)$

- If $X \sim \mathrm{K}(a, b)$, then $X \sim \operatorname{GB} 1(a, 1,1, b)$,

where GB1 stands for the generalized beta distribution of the first kind.

In this article we consider two independent (or sub-independent) Kumaraswamy random variables with appropriate parameters and study the convolution of their distributions (i.e., sum, difference, product and ratio). Also, we discuss some real life scenarios in which such convolutions might be an attractive model. This is the major contribution of this article. We list in sequel, the scenarios where sums, differences, products and ratios of two independent Kumaraswamy might be useful.

- Situations in which Kumaraswamy sums will be useful:

- Length or weight of a chain with $n$ links.

- Electric resistance of a series circuit.

- The total life length of $n$ products, where the next one is put on operation after failure of the preceding one.

In each of these cases, individual items (for example $X_{i}$ denoting individual links for the first example, individual resistance for the second example and individual life length for the third example) can be considered as having univariate Kumaraswamy densities.

- Situations in which Kumaraswamy differences will be useful:

- Let us suppose that in a certain mass-produced assembly, a $5 \mathrm{~cm}$ shaft must slide into a cylindrical sleeve. Shafts are manufactured whose diameter $X_{1}$ follows a Kumaraswamy distribution and cylindrical sleeves are manufactured whose internal diameter $X_{2}$ follows another Kumaraswamy distribution. Assembly is performed by selecting a shaft and a cylindrical sleeve at random. Suppose our interest is the following: In what proportion of cases will it be impossible to fit the selected shaft and cylindrical sleeve together. Clearly, the shaft and cylindrical sleeve will fit together only if the diameter of the shaft is smaller than the internal diameter of the cylindrical sleeve. We need the difference of the two random variables $X_{2}$ and $X_{1}$ to be greater than zero. We can take the difference $X_{2}-X_{1}$ and find its distribution.

- Situations in which Kumaraswamy product will be useful:

We cite a real life application below: 
- An observer's information about a classical system is captured by the observation that he/she assigns to it. It is a legitimate assumption that the two observers do not have to have the same information about the classical system. Next, if two observers have obtained their information about the system independently, then clearly together they have gathered more information about the system than each has individually. A natural question then is: is it possible to come up with a single observation which embodies their combined information? An answer to this question may be provided by considering distribution of the product of the two independent random variables.

* In evaluating the revenue from holding an asset defined by initial investment $\left(X_{1}\right) \times$ net return $\left(X_{2}\right)$.

* Consider the portfolio value accumulation scenario: A portfolio with current value $X_{1}$ will become $X_{1}\left(1+X_{2}\right)$ after some period, here $X_{2}$ is the interest rate and $X_{1}$ and $X_{2}$ are independent.

* ARCH models in time series studies.

* Number of cancer cells in tumor biology.

We believe that the above list of real life scenarios merits for the study of the distribution of the ratio $\frac{X_{1}}{X_{2}}$ and of the product $X_{1} X_{2}$ when the random variables $X_{1}$ and $X_{2}$ are independent and each follow a univariate Kumaraswamy distribution with the density function in (1.1).

The rest of the paper is organized as follows: In Section 1, we discuss the distribution of the sum, difference, product and the ratio. Section 2 deals with the distribution (as in Section 1) under non standard Kumaraswamy univariate distributions. In Section 3, we present some ideas of constructing bivariate Kumaraswamy distribution via copula and possible extension to the multivariate case. In Section 4, some concluding remarks are provided.

For the derivation of the distributions of the sum $S=X_{1}+X_{2}$, and of the difference $D=X_{1}-X_{2}$, the assumption of independence is not needed, a much weaker concept called sub-independence (defined below) can replace that of independence.

For the sake of completeness we will state below a few definitions related to the concept of subindependence. The concept of sub-independence is stated as follows: The $r v^{\prime} s$ (random variables) $X$ and $Y$ with $c d f^{\prime} s F_{X}$ and $F_{Y}$ are s.i. (sub-independent) if the $c d f$ of $X+Y$ is given by

$$
F_{X+Y}(z)=\left(F_{X} * F_{Y}\right)(z)=\int_{\mathbb{R}} F_{X}(z-y) d F_{Y}(y), \quad z \in \mathbb{R},
$$

or equivalently if and only if

$$
\varphi_{X+Y}(t)=\varphi_{X, Y}(t, t)=\varphi_{X}(t) \varphi_{Y}(t), \text { for all } t \in \mathbb{R},
$$

where $\varphi_{X}, \varphi_{Y}, \varphi_{X+Y}$ and $\varphi_{X, Y}$ are $c f^{\prime} s$ (characteristic functions) of $X, Y, X+Y$ and $(X, Y)$, respectively.

The equations (1.3) and (1.4) above are in terms of $c d f$ and $c f$. The definition of subindependence in terms of events, similar to that of independence, is as follows.

We observe that the half-plane $H=\{(x, y): x+y<0\}\left(\subseteq \mathbb{R}^{2}\right)$ can be written as a countable disjoint union of rectangles:

$$
H=\bigcup_{i=1}^{\infty} E_{i} \times F_{i}
$$


where $E_{i}$ and $F_{i}$ are intervals. Now, let $(X, Y): \Omega \rightarrow \mathbb{R}^{2}$ be a continuous random vector and for $c \in \mathbb{R}$, let

$$
A_{c}=\{\omega \in \Omega: X(\omega)+Y(\omega)<c\}
$$

and

$$
A_{i}^{(c)}=\left\{\omega \in \Omega: X(\omega)-\frac{c}{2} \in E_{i}\right\}, B_{i}^{(c)}=\left\{\omega \in \Omega: Y(\omega)-\frac{c}{2} \in F_{i}\right\} .
$$

Definition 1.1. The continuous $r v^{\prime} s X$ and $Y$ are s.i. if for every $c \in \mathbb{R}$

$$
P\left(A_{c}\right)=\sum_{i=1}^{\infty} P\left(A_{i}^{(c)}\right) P\left(B_{i}^{(c)}\right)
$$

Remark 1.1. (a) The representation (1.4) can be extended to the multivariate case as well.

(b) For a detailed treatment of the concept of sub-independence, please refer to Hamedani(2013) [1].

Consider the distribution of the sum $S=X_{1}+X_{2}$ when the $r v^{\prime} s X_{1}$ and $X_{2}$ are s.i., then $p d f$ of $S$ is the convolution of the $p d f^{\prime} s$ of $X_{1}$ and $X_{2}$, which is given by

$$
f_{S}(s)=\left\{\begin{array}{lll}
\int_{0}^{s} f_{X_{1}}(t) f_{X_{2}}(s-t) d t, & \text { if } \quad 0<s<1 \\
\int_{0}^{2-s} f_{X_{1}}(s-1+t) f_{X_{2}}(1-t) d t, & \text { if } \quad 1<s<2
\end{array} .\right.
$$

The distribution of the difference $D=X_{1}-X_{2}$ when the $r v^{\prime} s X_{1}$ and $-X_{2}$ are s.i., has a $p d f$ which is the convolution of the $p d f^{\prime} s$ of $X_{1}$ and $-X_{2}$, given by

$$
f_{D}(d)= \begin{cases}\int_{0}^{1+d} f_{X_{1}}(t) f_{X_{2}}(t-d) d t, & \text { if }-1<d<0 \\ \int_{0}^{1-d} f_{X_{1}}(d+t) f_{X_{2}}(t) d t, & \text { if } 0<d<1\end{cases}
$$

Now, assuming that the $r v^{\prime} s X_{1}$ and $X_{2}$ are independent, then $p d f^{\prime} s$ of $P=X_{1} X_{2}$ and of $R=\frac{X_{2}}{X_{1}}$ are given, respectively, by

$$
f_{P}(p)=\int_{p}^{1} \frac{f_{X_{1}}(t)}{t} f_{X_{2}}\left(\frac{p}{t}\right) d t, \quad 0<p<1
$$

and

$$
f_{R}(r)= \begin{cases}\int_{0}^{1} t f_{X_{1}}(t) f_{X_{2}}(r t) d t, & \text { if } \quad r \leq 1 \\ \int_{0}^{1 / r} t f_{X_{1}}(t) f_{X_{2}}(r t) d t, & \text { if } \quad r>1\end{cases}
$$

\section{Kumaraswamy Sums, Differences, Products and Ratios}

In this section we consider explicit expressions for the pdfs of $S, D, P$ and $R$ respectively. Note that for $S$ and $D$, we employ the concept of sub-independence. 


\subsection{Explicit expression for the pdf of the Sum (S)}

Theorem 2.1. For $0<s<2$, the pdf of $S$ will be

$$
f_{S}(s)= \begin{cases}\left(a_{2} b_{1} b_{2}\right) \sum_{j=0}^{\infty} \sum_{k=0}^{\infty}(-1)^{j+k}\left(\begin{array}{c}
b_{2}-1 \\
j
\end{array}\right)\left(\begin{array}{c}
a_{2}(1+j)-1 \\
k
\end{array}\right) s^{k} I_{s}^{a_{1}}\left(\frac{a_{2}(1+j)-1-k}{a_{1}}, b_{1}\right), & 0<s<1, \\
\left(a_{2} b_{1} b_{2}\right) \sum_{j=0}^{\infty} \sum_{k=0}^{\infty}(-1)^{j+k}\left(\begin{array}{c}
b_{1}-1 \\
j
\end{array}\right)\left(\begin{array}{c}
a_{1}(1+j)-1 \\
k
\end{array}\right) s^{k} \delta_{(s-1)^{a_{2}}}\left(\frac{a_{1}(1+j)+k}{a_{2}}+1, b_{2}\right), & 1<s<2,\end{cases}
$$

where $I_{x}(a, b)=\int_{0}^{x} u^{a-1}(1-u)^{b-1} d u, \delta_{x}(a, b)=\int_{x}^{1} u^{a-1}(1-u)^{b-1} d u$ are the lower and upper incomplete beta functions respectively, and $\left(\begin{array}{c}b_{2} \\ j\end{array}\right)=\frac{\left(b_{2}\right)_{(j)}}{j !}, \quad\left(b_{2}\right)_{(j)}=b_{2}\left(b_{2}-1\right) \cdots\left(b_{2}-1+j\right)$.

Proof. Consider the case $0<s<1$, we have from (1.6),

$$
\begin{aligned}
\int_{0}^{s} f_{1}(t) f_{2}(s-t) d t= & a_{1} b_{1} a_{2} b_{2} \int_{0}^{s} t^{a_{1}-1}\left(1-t^{a_{1}}\right)^{b_{1}-1}(s-t)^{a_{2}-1}\left[1-(s-t)^{a_{2}}\right]^{b_{2}-1} d t \\
= & a_{1} b_{1} a_{2} b_{2} \sum_{j=0}^{\infty}(-1)^{j}\left(\begin{array}{c}
b_{2}-1 \\
j
\end{array}\right) t^{a_{1}-1}\left(1-t^{a_{1}}\right)^{b_{1}-1}(s-t)^{a_{2}(1+j)-1} d t, \\
& \text { on using the generalized binomial expansion } \\
= & b_{1} a_{2} b_{2} \sum_{j=0}^{\infty}(-1)^{j}\left(\begin{array}{c}
b_{2}-1 \\
j
\end{array}\right) \int_{0}^{s^{a_{1}}}(1-u)^{b_{1}-1}\left[s-u^{1 / a_{1}}\right]^{a_{2}(1+j)-1} d u, \\
& \text { on using the transformation } u=t^{a_{1}} \\
= & b_{1} a_{2} b_{2} \sum_{j=0}^{\infty} \sum_{k=0}^{\infty}(-1)^{j+k}\left(\begin{array}{c}
b_{2} \\
j
\end{array}\right)\left(\begin{array}{c}
a_{2}(1+j)-1 \\
k
\end{array}\right) \int_{0}^{s^{a_{1}}}(1-u)^{b_{1}-1} u^{\frac{a_{2}(1+j)-1-k}{a_{1}}} d u \\
= & a_{2} b_{1} b_{2} \sum_{j=0}^{\infty} \sum_{k=0}^{\infty}(-1)^{j+k}\left(\begin{array}{c}
b_{2}-1 \\
j
\end{array}\right)\left(\begin{array}{c}
a_{2}(1+j)-1 \\
k
\end{array}\right) s^{k} I_{s^{a_{1}}}\left(\frac{a_{2}(1+j)-1-k}{a_{1}}+1, b_{1}\right) .
\end{aligned}
$$

The result for $1<s<2$ can be established similarly. Hence the proof.

Some representative density plots for $S$ is provided in Figures 1 and 2. The following observations can be made from these two graphs.

- When $b_{1}$ and $b_{2}$ are kept fixed, if all other possible combinations of the first two shape parameters (i.e., $a_{1}$ and $a_{2}$ ), the density appears to be slightly left-skewed (except for the case $a_{1}=a_{2}$, in which it is approximately symmetric, as expected) (see Figure 1).

- When $a_{1}$ and $a_{2}$ are kept fixed, if all other possible combinations of the second two shape parameters (i.e., $b_{1}$ and $b_{2}$ ), the density appears to be slightly right-skewed (except for the case $b_{1}=b_{2}$, in which it is approximately symmetric, as expected) (see Figure 2).

As a consequence, in order to model bounded risks (with a proper modification, if required, to be in the interval $[0,1]$ ) with a right skewed data, one might consider the scenario of changing the second shape parameter and keeping the first shape parameter fixed and vice versa for the left skewed data. 


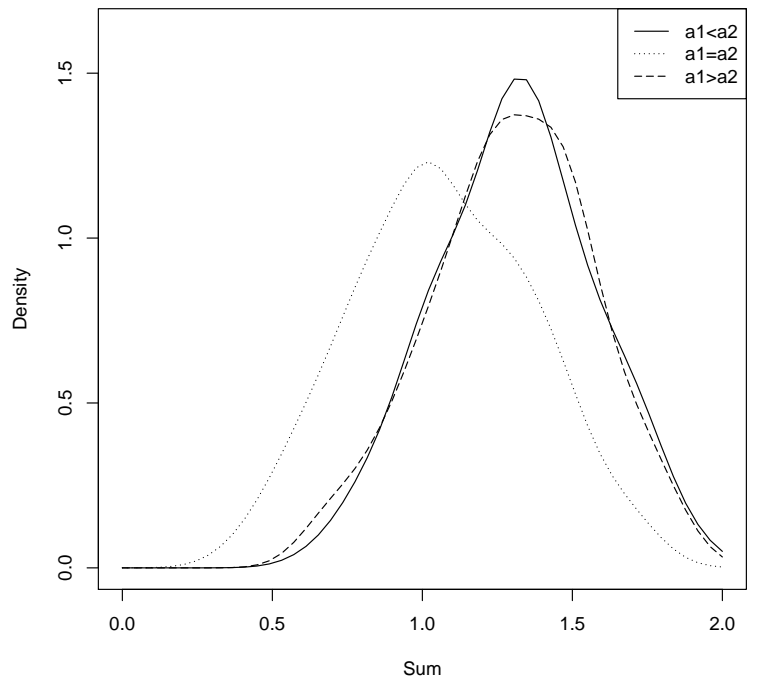

Fig. 1. Density plot of $S$ with $b_{1}, b_{2}$ fixed.

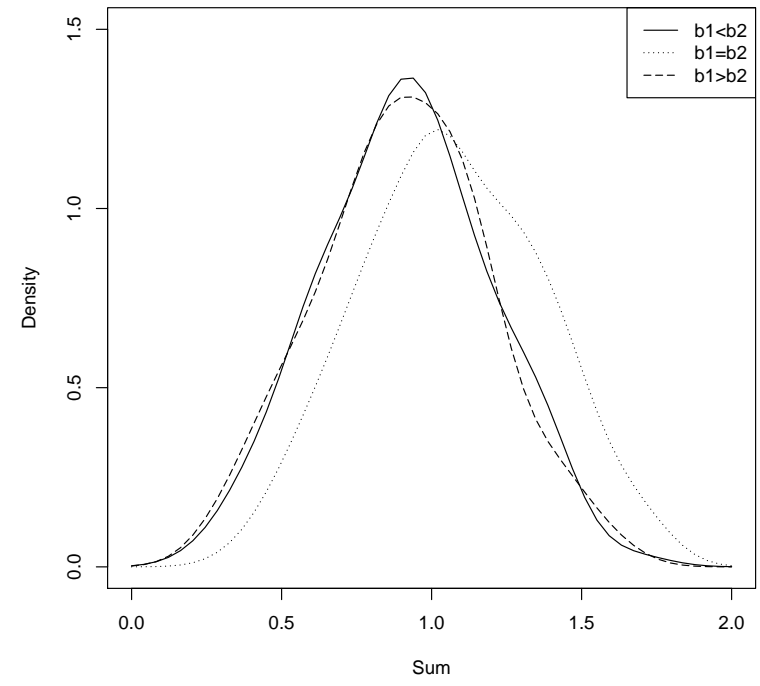

Fig. 2. Density plot of $S$ with $a_{1}, a_{2}$ fixed.

\subsection{Explicit expression for the pdf of the Difference (D)}

Theorem 2.2. For $-1<d<1$, the pdf of $D$ will be

$$
f_{D}(d)=\left\{\begin{array}{r}
\left(a_{2} b_{1} b_{2}\right) \sum_{j=0}^{\infty} \sum_{k=0}^{\infty}(-1)^{j+k}\left(\begin{array}{c}
b_{2}-1 \\
j
\end{array}\right)\left(\begin{array}{c}
a_{2}(1+j)-1 \\
k
\end{array}\right) d^{k} I_{(1+d)^{a_{1}}}\left(\frac{a_{2}(1+j)-1-k}{a_{1}}+1, b_{1}\right), \\
-1<d<0, \\
\left(a_{2} b_{1} b_{2}\right) \sum_{j=0}^{\infty} \sum_{k=0}^{\infty}(-1)^{j+k}\left(\begin{array}{c}
b_{1}-1 \\
j
\end{array}\right)\left(\begin{array}{c}
a_{1}(1+j)-1 \\
k
\end{array}\right) d^{a_{1}\left(1+j_{1}\right)-1-k} \delta_{(1-d)^{a_{2}}}\left(\frac{k}{a_{2}}+1, b_{2}\right), \\
0<d<1 .
\end{array}\right.
$$

Proof. Similar to that of Theorem 2.1.

Some representative density plots of $D$ are provided in Figures 3 and 4. The following observations can be made from these two graphs.

- When $b_{1}$ and $b_{2}$ are kept fixed, the density appears to be slightly right skewed, if $a_{1}<a_{2}$ and left-skewed if $a_{1}>a_{2}$, with the obvious symmetric scenario is which $a_{1}=a_{2}$ (see Figure $3)$.

- When $a_{1}$ and $a_{2}$ are kept fixed, the density appears to be slightly left -skewed, if $b_{1}<$ $b_{2}$. Noticeably, the density appears to be approximately symmetric in the remaining two parametric configurations (i.e., in $b_{1}=b_{2}$ and $b_{1}>b_{2}$ ) (see Figure 4).

It appears that for the difference, the form of the density is very sensitive to any change in the first shape parameters (i.e., $a_{1}$ and $a_{2}$ ). 


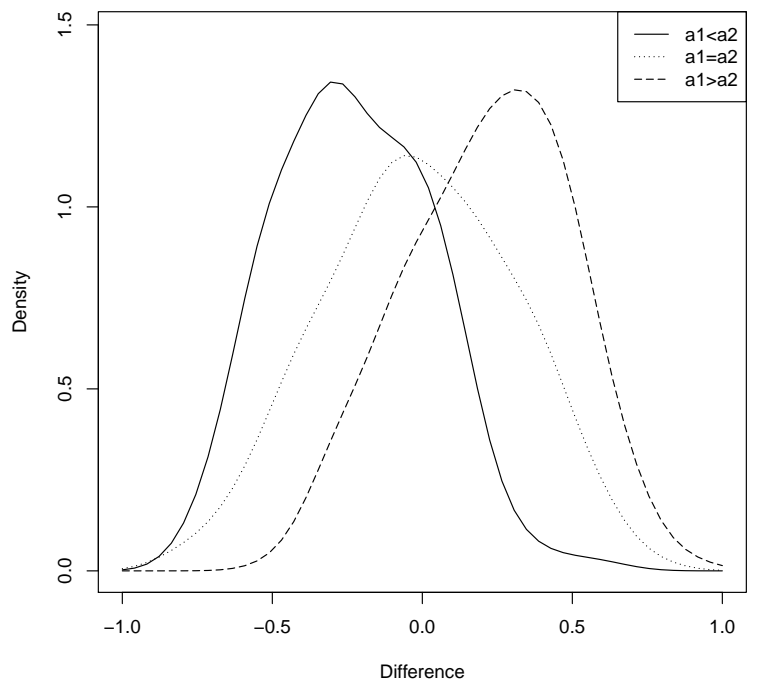

Fig. 3. Density plot of $D$ with $b_{1}, b_{2}$ fixed.

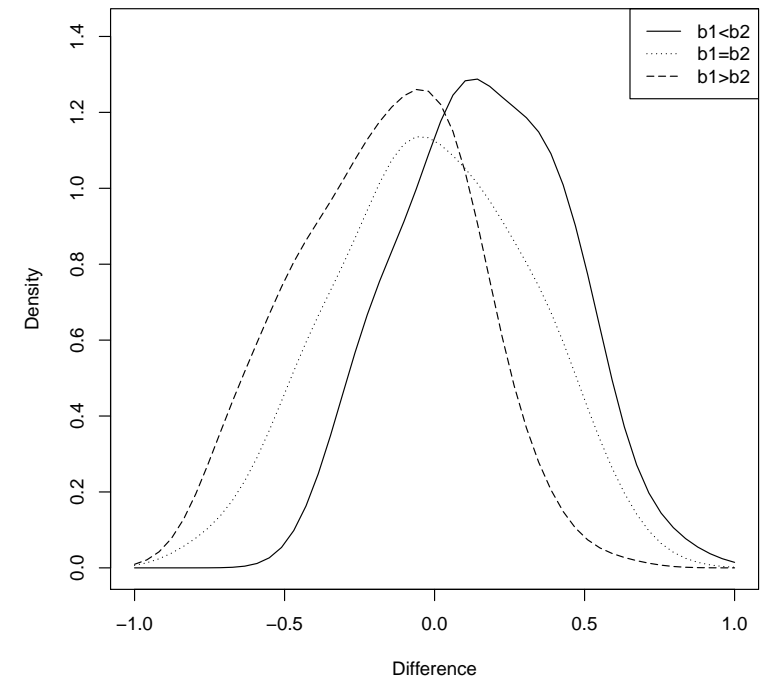

Fig. 4. Density plot of $D$ with $a_{1}, a_{2}$ fixed.

\subsection{Explicit expression for the pdf of the Product $(P)$}

Theorem 2.3. For $0<p<1$, the pdf of $P$ will be

$$
f_{P}(p)=\left(a_{2} b_{1} b_{2}\right) \sum_{j=0}^{\infty}(-1)^{j}\left(\begin{array}{c}
b_{2}-1 \\
j
\end{array}\right) p^{a_{2}(1+j)-1} \delta_{(p)^{a_{1}}}\left(\frac{2-a_{2}(1+j)}{a_{1}}+1, b_{1}\right)
$$

Proof. From (1.8), we can write

$$
\begin{aligned}
f_{P}(p)= & \left(a_{1} a_{2} b_{1} b_{2}\right) \int_{p}^{1} t^{a_{1}}\left[\left(1-t^{a_{1}}\right)^{b_{1}-1}\right]\left[\left(\frac{p}{t}\right)^{a_{2}-1}\left(1-\left(\frac{p}{t}\right)^{a_{2}}\right)^{b_{2}-1}\right] d t \\
= & \left(a_{1} a_{2} b_{1} b_{2}\right) \sum_{j=0}^{\infty}(-1)^{j}\left(\begin{array}{c}
b_{2}-1 \\
j
\end{array}\right) p^{a_{2}(1+j)-1} \int_{p}^{1} t^{a_{1}+1-a_{2}(1+j)}\left(1-t^{a_{1}}\right)^{b_{1}-1} d t, \\
& \text { again using the binomial series expansion } \\
= & \left(a_{2} b_{1} b_{2}\right) \sum_{j=0}^{\infty}(-1)^{j}\left(\begin{array}{c}
b_{2}-1 \\
j
\end{array}\right) p^{a_{2}(1+j)-1} \delta_{(p)^{a_{1}}}\left(\frac{2-a_{2}(1+j)}{a_{1}}+1, b_{1}\right) .
\end{aligned}
$$

Hence the result.

Some representative density plots of $P$ are provided in Figures 5 and 6. From both of these plots it appears that regardless of the choice of the for four shape parameters, the density of $P$ is right skewed. 


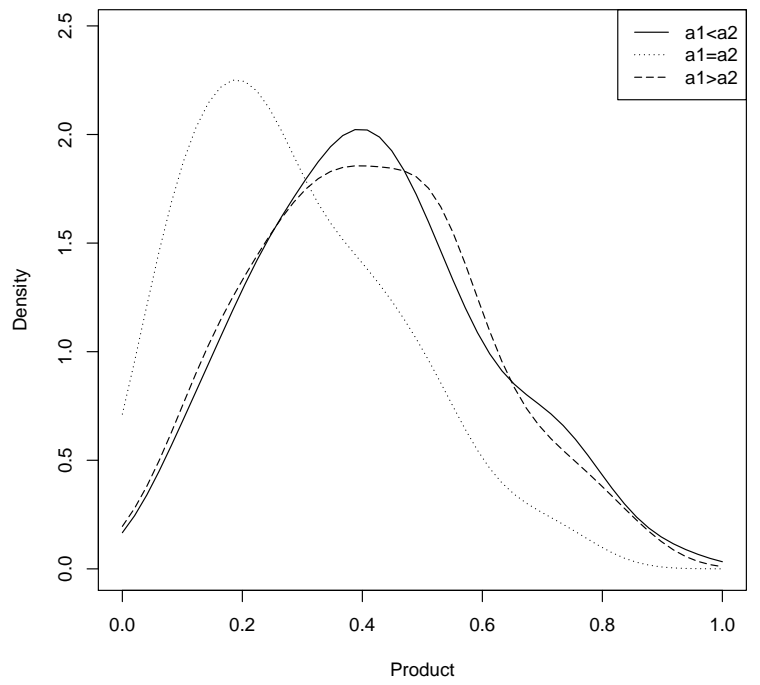

Fig. 5. Density plot of $P$ with $b_{1}, b_{2}$ fixed.

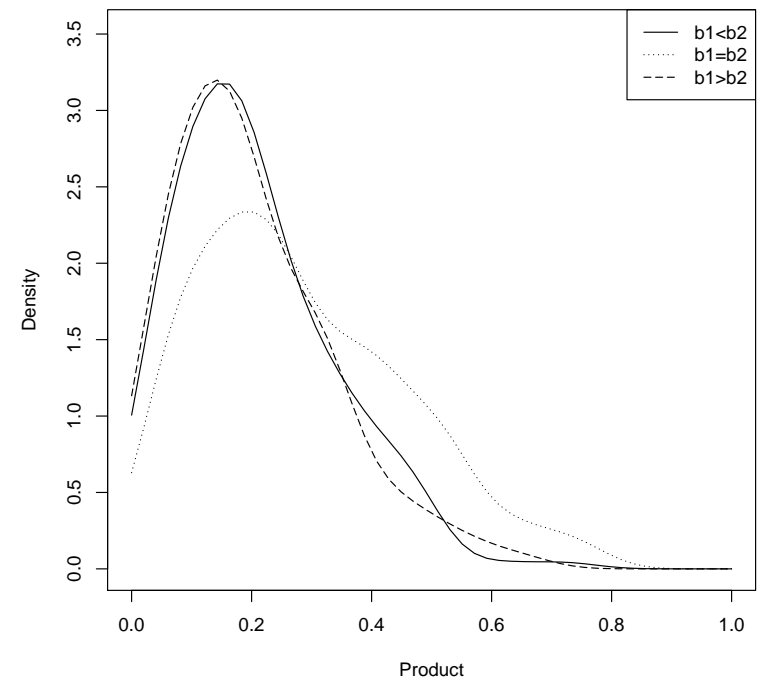

Fig. 6. Density plot of $P$ with $a_{1}, a_{2}$ fixed.

\subsection{Explicit expression for the pdf of the Ratio $(R)$}

Theorem 2.4. For $0<r<\infty$, the pdf of $R$ will be

$$
f_{R}(r)= \begin{cases}\left(a_{2} b_{1} b_{2}\right) \sum_{j=0}^{\infty}(-1)^{j}\left(\begin{array}{c}
b_{2}-1 \\
j
\end{array}\right) r^{2 a_{2}+j-1} B\left(\frac{2 a_{2}+j+1}{a_{1}}+1, b_{1}\right), & 0<r<1, \\
\left(a_{2} b_{1} b_{2}\right) \sum_{j=0}^{\infty}(-1)^{j}\left(\begin{array}{c}
b_{2}-1 \\
j
\end{array}\right) r^{2 a_{2}+j-1} I_{(1 / r)^{a_{1}}}\left(\frac{2 a_{2}+j+1}{a_{1}}+1, b_{1}\right), & 1<r<\infty .\end{cases}
$$

Proof. Let us consider the case $0<r \leq 1$. From (1.9), we can write

$$
\begin{aligned}
\int_{0}^{1} t \times f_{1}(t) f_{2}(r t) d t= & \left(a_{1} b_{1} a_{2} b_{2}\right) \int_{0}^{s} t \times t^{a_{1}-1}\left(1-t^{a_{1}}\right)^{b_{1}-1}(r t)^{a_{2}-1}\left[1-(r t)^{a_{2}}\right]^{b_{2}-1} d t \\
= & \left(a_{1} b_{1} a_{2} b_{2}\right) \sum_{j=0}^{\infty}(-1)^{j}\left(\begin{array}{c}
b_{2}-1 \\
j
\end{array}\right) r^{a_{2}-1} t^{a_{1}+a_{2}-1}\left(1-t^{a_{1}}\right)^{b_{1}-1}(r t)^{a_{2}+j} d t \\
& \text { on using the generalized binomial expansion } \\
= & \left(b_{1} a_{2} b_{2}\right) \sum_{j=0}^{\infty}(-1)^{j}\left(\begin{array}{c}
b_{2}-1 \\
j
\end{array}\right) r^{2 a_{2}+j-1} \int_{0}^{1} u^{\left(2 a_{2}+j\right) / a_{1}}[1-u]^{b_{1}-1} d u \\
& \text { on using the transformation } u=t^{a_{1}} \\
= & \left(a_{2} b_{1} b_{2}\right) \sum_{j=0}^{\infty}(-1)^{j}\left(\begin{array}{c}
b_{2}-1 \\
j
\end{array}\right) r^{2 a_{2}+j-1} B\left(\frac{2 a_{2}+j}{a_{1}}+1, b_{1}\right) .
\end{aligned}
$$

The result for $1<r<\infty$ can be established similarly. Hence the proof.

Note: If $b_{2}-1$ is an integer, the sum will stop at $b_{2}-1$.

Some representative density plots of $R$ are provided in Figures 7 and 8. From both these plots it 
appears that regardless of the choice of the for four shape parameters, the density of $R$ is strictly right skewed.

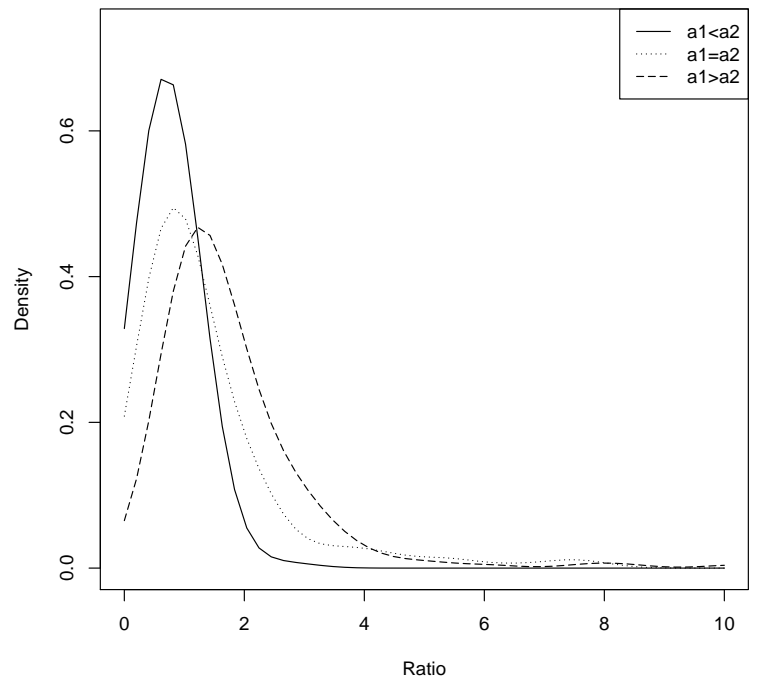

Fig. 7. Density plot of $R$ with $b_{1}, b_{2}$ fixed.

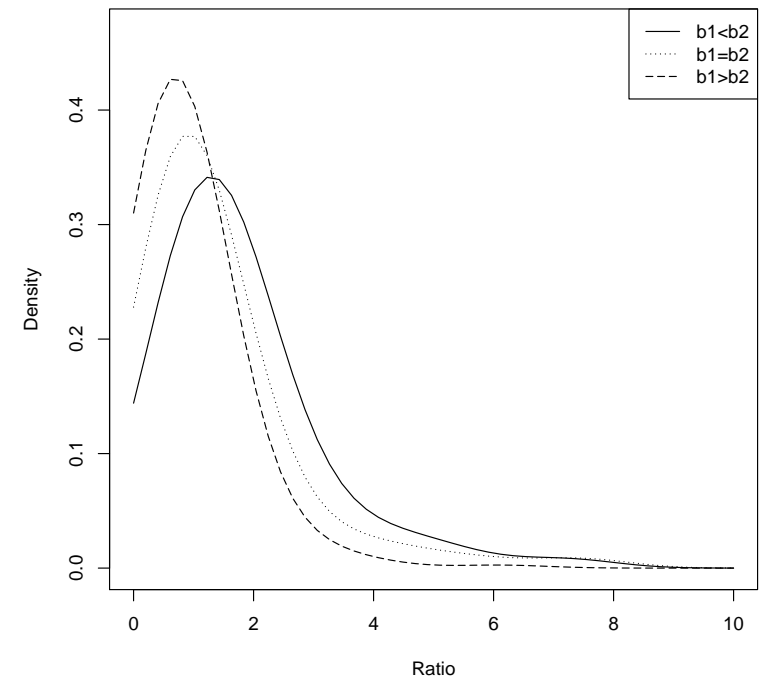

Fig. 8. Density plot of $R$ with $a_{1}, a_{2}$ fixed.

Remark 2.1. We have also provided some representative density plots (see Figures 9 and 10) for the density of $W=\frac{X_{1}}{X_{1}+X_{2}}$. It appears that when we consider different combinations of the first shape parameters (i.e., $a_{1}$ and $a_{2}$ ), keeping the second set of shape parameters fixed, the density is approximately symmetric. While for the other case, it is slightly left-skewed.

\section{Sum, Difference, Product and Ratio for non-central Kumaraswamy distribution}

In this section we will make frequent use of the following representation of a Kumaraswamy variable as a power of a Beta variable. If $Y \sim \operatorname{Bet} a(1, b)$, then $X=Y^{1 / a} \sim K(a, b)$. Next, we start with three non-central beta models (for details, see Nagar et al. (2013) [4]) and subsequently obtain the expression of the densities of Sum (S), Product (P) and the Ratio (R).

The non-central Type I beta distribution is given by

$$
f(u)=\frac{\exp (-\delta) u^{a-1}(1-u)^{b-1}}{B(a, b)}{ }_{1} F_{1}(b+1 ; b ; \delta(1-u)), 0<u<1,
$$

where ${ }_{1} F_{1}(a ; c ; z)=\sum_{j=0}^{\infty} \frac{\Gamma(c) \Gamma(a+j) z^{j}}{j ! \Gamma(a) \Gamma(c+j)}$.

The Type I beta distribution is well known in Bayesian methodology as a prior distribution on the success probability of a binomial distribution. Next, setting $a=1$ and then by making the transformation $X=U^{1 / \alpha}$, (for any $\alpha>0$ ), the corresponding non-central Kumaraswmy (Type I) 


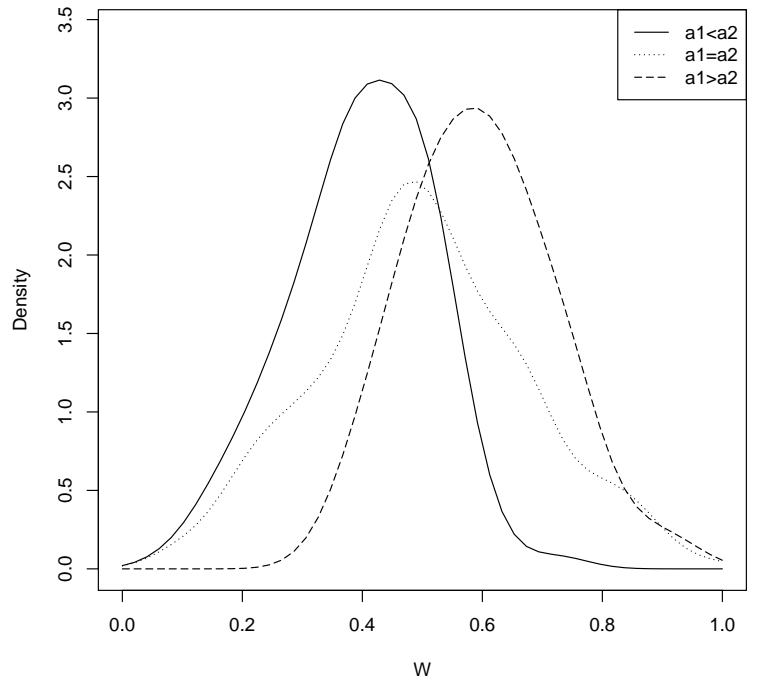

Fig. 9. Density plot of $W$ with $b_{1}, b_{2}$ fixed.

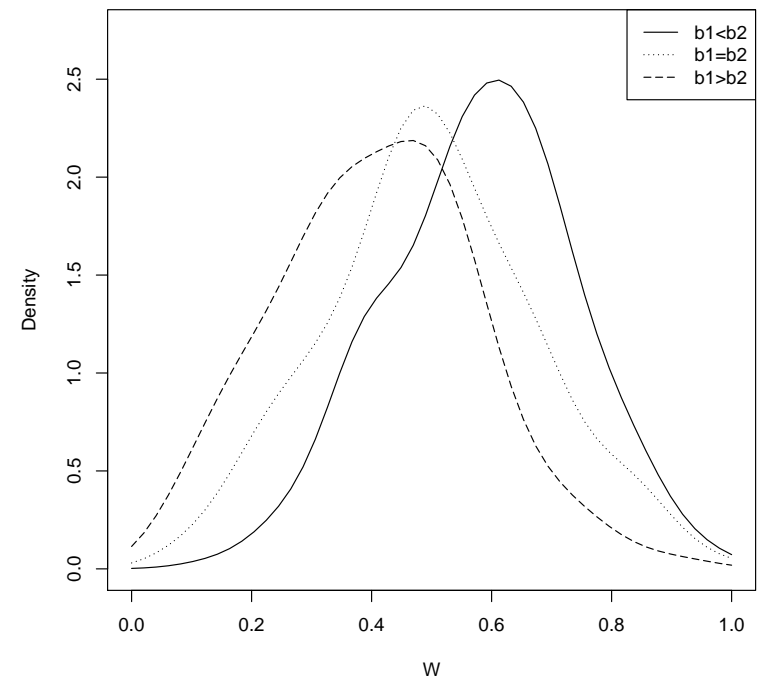

Fig. 10. Density plot of $W$ with $a_{1}, a_{2}$ fixed.

will be

$$
f(x)=b \alpha x^{\alpha-1} \exp (-\delta)\left(1-x^{\alpha}\right)^{b-1}{ }_{1} F_{1}\left(b+1 ; b ; \delta\left(1-x^{\alpha}\right)\right), 0<x<1 .
$$

For notational simplicity, henceforth we call the above distribution as $X \sim \operatorname{NCKW}($ TypeI $)(\alpha, \delta, b)$. Next, we have the following theorem:

Theorem 3.1. Suppose $X_{1} \sim N C K W($ TypeI $)\left(\alpha_{1}, \delta_{1}, b_{1}\right)$ and $X_{1} \sim N C K W(T y p e I)\left(\alpha_{2}, \delta_{2}, b_{2}\right)$ and they are sub-independent. Then for $0<s<2$, the pdf of $S$ will be

$$
f_{S}(s)= \begin{cases}\theta \sum_{j_{1}=0}^{\infty} \sum_{j_{2}=0}^{\infty} \sum_{j_{3}=0}^{\infty} \sum_{j_{4}=0}^{\infty}(-1)^{j_{3}+j_{4}}\left(\begin{array}{c}
b_{1}-1+j_{1} \\
j_{3}
\end{array}\right)\left(\begin{array}{c}
b_{2}-1+j_{2} \\
j_{4}
\end{array}\right) A_{1}, & 0<s<1, \\
\theta \sum_{j_{1}=0}^{\infty} \sum_{j_{2}=0}^{\infty} \sum_{j_{3}=0}^{\infty} \sum_{j_{4}=0}^{\infty}(-1)^{j_{3}+j_{4}}\left(\begin{array}{c}
b_{1}-1+j_{1} \\
j_{3}
\end{array}\right)\left(\begin{array}{c}
b_{2}-1+j_{2} \\
j_{4}
\end{array}\right)\left(\begin{array}{c}
b_{2}-1+j_{2} \\
j_{4}
\end{array}\right) A_{2}, & 1<s<2,\end{cases}
$$

where $\theta=\left(b_{1} b_{2} \alpha_{1} \alpha_{2} \exp \left(-\left(\delta_{1}+\delta_{2}\right)\right)\right)$,

$$
A_{1}=\left[\frac{\Gamma\left(b_{1}\right) \Gamma\left(b_{2}\right)\left(b_{1}+j_{1}\right)\left(b_{2}+j_{2}\right) \delta_{1}^{j_{1}} \delta_{2}^{j_{2}}}{j_{1} ! j_{2} !}\right]\left[\frac{\Gamma\left(\left(j_{3}+1\right) \alpha_{1}\right) \Gamma\left(\left(j_{4}+1\right) \alpha_{2}\right) s^{\alpha_{1}\left(j_{3}+1\right)+\alpha_{2}\left(j_{34}+1\right)-1}}{\Gamma\left(\left(j_{3}+1\right) \alpha_{1}+\left(j_{4}+1\right) \alpha_{2}\right)}\right],
$$


and

$$
\begin{aligned}
& A_{2}=s^{-2}(s-1)^{\alpha_{2}\left(j_{4}+1\right)}\left(\frac{s-1}{s}\right)^{\alpha_{2}\left(-\left(j_{4}+1\right)\right)} \times\left[\frac{s_{2} F_{1}\left(\left(j_{3}+1\right) \alpha_{1},-\left(j_{4}+1\right) \alpha_{2} ;\left(j_{3}+1\right) \alpha_{1}+1 ; \frac{1}{s}\right)}{\alpha_{1}\left(j_{3}+1\right)}\right. \\
& \left.+\frac{{ }_{2} F_{1}\left(\left(j_{3}+1\right) \alpha_{1}+1,1-\left(j_{4}+1\right) \alpha_{2} ;\left(j_{3}+1\right) \alpha_{1}+2 ; \frac{1}{s}\right)}{\alpha_{1}\left(j_{3}+1\right)+1}\right] \\
& -(s-1)^{\alpha_{1}\left(j_{3}+1\right)}\left(\frac{1}{s}\right)^{\alpha_{2}\left(-\left(j_{4}+1\right)\right)} \times\left[\frac{s_{2} F_{1}\left(\left(j_{3}+1\right) \alpha_{1},-\left(j_{4}+1\right) \alpha_{2} ;\left(j_{3}+1\right) \alpha_{1}+1 ; \frac{s-1}{s}\right)}{\alpha_{1}\left(j_{3}+1\right)}\right. \\
& \left.+\frac{(s-1){ }_{2} F_{1}\left(\left(j_{3}+1\right) \alpha_{1}+1,1-\left(j_{4}+1\right) \alpha_{2} ;\left(j_{3}+1\right) \alpha_{1}+2 ; \frac{s-1}{s}\right)}{\alpha_{1}\left(j_{3}+1\right)+1}\right] \text {. }
\end{aligned}
$$

Proof. Similar to that of Theorem 2.1.

Theorem 3.2. For $-1<d<1$, the $p d f$ of $D$ will be

$$
f_{D}(d)=\left\{\begin{array}{l}
\theta \sum_{j_{1}=0}^{\infty} \sum_{j_{2}=0}^{\infty} \sum_{j_{3}=0}^{\infty} \sum_{j_{4}=0}^{\infty}(-1)^{j_{3}+j_{4}}\left(\begin{array}{c}
b_{1}-1+j_{1} \\
j_{3}
\end{array}\right)\left(\begin{array}{c}
b_{2}-1+j_{2} \\
j_{4}
\end{array}\right) B_{1}, \quad-1<d<0, \\
\theta \sum_{j_{1}=0}^{\infty} \sum_{j_{2}=0}^{\infty} \sum_{j_{3}=0}^{\infty} \sum_{j_{4}=0}^{\infty}(-1)^{j_{3}+j_{4}}\left(\begin{array}{c}
b_{1}-1+j_{1} \\
j_{3}
\end{array}\right)\left(\begin{array}{c}
b_{2}-1+j_{2} \\
j_{4}
\end{array}\right) B_{2}, \quad 0<d<1,
\end{array}\right.
$$

where

$$
B_{1}=(-d-1)^{\alpha_{1}\left(-j_{3}-1\right)}(-d)^{\alpha_{1}\left(j_{3}+1\right)}(-d)^{\alpha_{2}\left(j_{4}+1\right)-1}(d+1)^{\alpha_{1}\left(j_{3}+1\right)} B_{1+\frac{1}{d}}\left(\left(j_{3}+1\right) \alpha_{1},\left(j_{4}+1\right) \alpha_{2}\right),
$$

and

$B_{2}=(-d-1)^{\alpha_{1}\left(-j_{3}-1\right)}(d-1)^{\alpha_{2}\left(-j_{4}-1\right)} d^{\alpha_{1}\left(j_{3}+1\right)-1}((1-d) d)^{\alpha_{2}\left(j_{4}+1\right)} B_{\frac{d-1}{d}}\left(\left(j_{4}+1\right) \alpha_{2},\left(j_{3}+1\right) \alpha_{1}\right)$.

Proof. Similar to that of Theorem 2.2.

Theorem 3.3. For $0<p<1$, the pdf of $P$ will be $f_{P}(p)=\theta \sum_{j_{1}=0}^{\infty} \sum_{j_{2}=0}^{\infty} \sum_{j_{3}=0}^{\infty} \sum_{j_{4}=0}^{\infty}(-1)^{j_{3}+j_{4}}\left(\begin{array}{c}b_{1}-1+j_{1} \\ j_{3}\end{array}\right)\left(\begin{array}{c}b_{2}-1+j_{2} \\ j_{4}\end{array}\right) \frac{p^{\alpha_{2}\left(j_{4}+1\right)}-p^{\alpha_{1}\left(j_{3}+1\right)+1}}{p\left(\alpha_{1}\left(j_{3}+1\right)-\alpha_{2}\left(j_{4}+1\right)+1\right)}$, provided $\alpha_{1}-\alpha_{2}-1 \geq 0$.

Theorem 3.4. For $0<r<\infty$, the pdf of $R$ will be

$$
f_{R}(r)=\left\{\begin{array}{r}
\theta \sum_{j_{1}=0}^{\infty} \sum_{j_{2}=0}^{\infty} \sum_{j_{3}=0}^{\infty} \sum_{j_{4}=0}^{\infty}(-1)^{j_{3}+j_{4}}\left(\begin{array}{c}
b_{1}-1+j_{1} \\
j_{3}
\end{array}\right)\left(\begin{array}{c}
b_{2}-1+j_{2} \\
j_{4}
\end{array}\right)\left[\begin{array}{r}
r^{\alpha_{2}\left(j_{4}+1\right)-1} \\
0<r<1,
\end{array}\right], \\
\theta \sum_{j_{1}=0}^{\infty} \sum_{j_{2}=0}^{\infty} \sum_{j_{3}=0}^{\infty} \sum_{j_{4}=0}^{\infty}(-1)^{j_{3}+j_{4}}\left(\begin{array}{c}
b_{1}-1+\alpha_{2}\left(j_{4}+1\right)-1 \\
j_{3}
\end{array}\right)\left(\begin{array}{c}
b_{2}-1+j_{2} \\
j_{4}
\end{array}\right)\left[\begin{array}{r}
\left(\frac{1}{r}\right)^{\alpha_{1}\left(j_{3}+1\right)} \\
\alpha_{1}\left(j_{3}+1\right)+\alpha_{2}\left(j_{4}+1\right)-1
\end{array}\right], \\
1<r<\infty .
\end{array}\right.
$$


Remark 3.1. One can also obtain in a similar fashion, the distribution of the Sum, Difference, Product, Ratios from the following two other non-central Kumaraswamy distributions:

- Non central Kumaraswamy (Type II) given by

$$
f(x)=b \exp (-\delta) x^{\alpha}\left(1+x^{\alpha}\right)^{-(b+1)}{ }_{1} F_{1}\left(b+1 ; b ; \frac{\delta}{\left(1+x^{\alpha}\right)}\right), 0<x<1 .
$$

- Non central Kumaraswamy (Type III) given by

$$
f(x)=\frac{b 2^{a} \exp (-\delta) x^{\alpha-1}\left(1-x^{\alpha}\right)^{b-1}}{\left(1+x^{\alpha}\right)^{b+1}}{ }_{1} F_{1}\left(b+1 ; b ; \frac{\delta\left(1-x^{\alpha}\right)}{\left(1+x^{\alpha}\right)}\right), 0<x<1 .
$$

\section{Conclusion}

In this article, by using the traditional method of transformation of variables, we have obtained probability density functions of sum, difference, product of two independent random variables both having regular Kumaraswamy and also non-central Kumaraswamy (Type I) distribution. Furthermore, in recent times, the construction of bivariate and multivariate Kumaraswamy distributions has received a significant amount of attention. Possible future works that can be done based on this article are as follows:

- Possible applications of independent Kumarswamy sums, products and ratios.

- Construction of Kumaraswamy sums, products etc. via dependent set up.

- A comparison study between beta distribution Sums, Products, Ratios and Differences etc.

Future efforts will be made to address these points and subsequently be reported elsewhere.

\section{References}

[1] G.G. Hamedani, Sub-Independence: An Expository Perspective. Communications in Statistics: Theory \& Methods, 42 (2013) 3615-3638

[2] M.C. Jones, A beta-type distribution with some tractability advantages. Statistical Methodology, 6 (2009) 70-81.

[3] P. Kumaraswamy, A generalized probability density function for double- bounded random processes. Journal of Hydrology, 46 (1980) 79-88.

[4] D.K. Nagar and Y.A. Ramirez-Vanegas, Distributions of Sum, Difference, Product and Quotient of Independent Non-central Beta Type 3 Variables. British Journal of Mathematics \& Computer Science, 3 (2013) 12-23. 